\title{
EL LENCA DE CHILANGA: LÉXICO
}

\author{
Ximena del Río Urrutia
}

\begin{abstract}
En este artículo presentamos el léxico recopilado del lenca salvadoreño en las fuentes escritas de Lehman (1920) y Campbell (1976).
\end{abstract}

\section{RESUMEN}

This article offers the vocabulary of Salvadorian Lenca extracted from the data in Lehman (1920) and Campbell (1976).

\section{Léxico español-lenca}

El siguiente léxico registra todos los términos que aparecen en Lehman (1920), incluyendo Peccorini (1911) y Sapper (1895); además del trabajo de Campbell (1976).

Junto con mi transcripción fonológica, se transcriben los términos con indicación de la fuente de donde se obtuvieron. Para los verbos, se da como forma base la primera persona singular del presente. En caso de no ser esto posible, se registra la forma que existe en los datos acompañada de su traducción. Además, se incluye un anexo con la matriz flexiva de los verbos, así como algunas indicaciones morfofonológicas sobre ellos. Cuando se han separado elementos de una forma, esto se indica poniendo un guión en lugar de ellos.

\section{A}

1. Abeja

2. Abrir

3. Abuela

4. Abuelo

5. Acabar

6. Achote

7. Adiós japifko

inkolo-

teteh

Joko

inarji-

awalk'e

wumpala ÿáp-ísko (Leh) in'gōlokón (Leh) 'abro' u-d̂̂d̂̂X-na (Leh) u-šogốna (Leh) ínartšikámba (Leh) auálk' ē (Leh) ùumbāla (Leh) 
8. Agua

9. Aguacate

10. Aguamatate

11. Aguardar

12. Ahora

13. Ala

14. Alacín

15. Alcanzar

16. Aleta

17. Algo

18. Algodón

19. Aliento

20. Almuerzo

21. Alto

22. Allá, allí

23. Amargo

24. Amarillo

25. Amarrar

26. Anciana (cf. abuela)

27. Anciano

28. Anguila

29. Anona blanca

30. Añil

31. Año

32. Aquí

33. Arado

34. Araña wal

1. ưál (Leh)

2. wal (Pec)

3. wal (Cam)

Jik'a

k'el

mapoli-

infalin

Juma

sojojo

mats'otap-

$\mathrm{kal}$

kasa

ts'uj

ink'ala

almwersu

kot'a

inum

tonis-ke-pa

$\mathrm{ku}$

war-

teteh

koko

ulum

jumi

mostasa

pulan

nanum

Jimkum-lawa

katu
1. šik'a šsia (Cam)

2. śik'à-žéla (Leh)

(žela 'maduro')

3. shía (Pec)

k'èl (Leh)

māpờikón

in`žấlin' (Leh)

1. šúma(Leh)

2. iyúma (Pec)

sỏÿóÿo (Leh)

mats-otapon (Leh)

í-gàl (Leh)

kāsa (Leh)

ts'ūi (Leh)

in'k'ấla (Leh)

almwersu-na (Cam)

1. ủakặtă-ba (Leh)

2. waco't'a (Pec)

3. huatara-got'a (Pec) 'horcón alto'

1. ínum (Leh)

2. inúnd-ra (Leh)

tonin'-gê-ba (Leh)

(lit. 'no es dulce')

1. kū (Leh)

2. cúna $(\mathrm{Pec})$

gủarón (Leh)

tâde (Leh)

1. kốgo (Leh)

2. cogo (Sap)

1. uluN-na lum-na (Can)

2. ủlùm (Leh)

3. ulumna (Pec)

ÿúmi (Leh)

mōstấsā (Leh)

pūlàn (Leh)

nấnum (Leh)

shímgum-láwa (Pec)

kấdū (Leh) 
35. Árbol

36. Arcilla

37. Arco

38. Ardilla

39. Arena

40. Asa

(cf. oreja)

41. Atar

42. Ayer

43. Ayote

44. Bagre

45. Bajo

46. Bailar

47. Bailarín

48. Baile

49. Banca (de madera) (Cf. árbol)

50. Bañar (se)

51. Barba

52. Barbona (planta)

53. barrer

54. Barriga

55. Barro

56. Beber

57. Besar

58. Blanco

59. Boca

60. Bolillo para tambor sun

ts'akaja

t'ilkin

Jurih

milea

tokoro

Ver amarrar

summan

ts'iwan

osoke

ruku

uli- ul

ulkapara

ulkin

sun

twa-

Jumah

ifu-majila

t'ap-

k'omo

Ver Arcilla

tali- tal-

$\int \mathrm{u} \int \mathrm{i}-$

Joko

in-ts'ats'a

Ver Árbol
1. suN (Cam)

2. sún (Leh)

3. zun (Alc)

ts'agáÿa (Leh)

t'íškin' (Leh)

1. šurih (Cam)

2. shuri (Pec)

3. súli (Leh)

mižéa (Leh)

ídogốro (Leh)

súmàn, (Leh)

1. ts'iwaN (Cam)

2. ts'íluăn` (Leh)

ōzogé (Leh)

warrugú $(\mathrm{Pec})$

huatara-rúgu (Pec)

'horcón bajo'

ūlikón’ (Leh)

úlgabắra (Leh)

úlgin` (Leh)

sūùn (Leh)

1. tūakớn` (Leh)

2. tuacón (Pec)

1. mấ-šúma -na (Leh)

2. idzu-mazila (Leh)

îdžū-māžíila (Leh)

t'ápkớn (Leh)

mā-k'ơmố-na (Leh)

1. talikón' (Leh)

2. talicón (Pec)

ižušíga (Leh)

¡Béselo!

1. šoko šogo (Cam)

2. shógo (Pec)

3. žōgo sắgo (Leh)

1. ints'ats'a (Cam)

2. ín-ts'ăts'a (Leh)

3. iu-tsa-tsa (Pec)

(Cf ts'ats'a 'piel') 
61. Bolsetoro

(avispa)

62. Bonito

63. Borracho

64. Bostezar

65. Brazo

(cf. atleta)

66. Brincar

67.¡Buenas tardes!

68. Bueno

69. ¡Buenos días!

70. Buho

71. Caballo

72. Cabello

73. Cabeza

74. Cacao

75. Caer

76. Caites

77. Calor

78. Calabaza

79. Caliente

80. Caliente, estar

81. Calvo

82. Camarón

83. Caminar

(cf. ir)

84. Camino

85. Camote kawjiri

c'' is

waltal

inkala-

kal

pi-

samaripo

Jam

sela kapu

iskirip'upu

kajo

alah

oso

k'akaw

pit-

watih

Ver sol

malu

a $a$

afan-

kolo

sisis

k'iN

kamoti kaužííri (Leh)

tš'ís (Leh)

waldal (Pec)

in'kălakámba (Leh)

(tal vez ink'ala 'respirar', transcribió la glotalización)

1. ma-gál-na (Leh)

2. cal (Pec)

pīrkokón' (Leh)

$1^{\mathrm{a}}$. S. Futuro

samarípo (Leh)

1. šăm (Leh)

2. shámba $(\mathrm{Pec})$

sæélakấpu (Leh)

ískri-p'up'u (Leh)

kàyo (Leh)

1. alah (Cam)

2. pi-ála pi-alá $\chi$-na (Leh)

3. ála (Pec)

1. pi-ozô-na (Leh)

2. u-yoşo-na (Cam)

3. iodso (Pec)

4. yótzo (Sap)

1. k’ấgau (Leh)

2. cacao (Pec)

pitón" (Leh)

1. watij (Pec)

2. u้ati u้atî́s-na (Leh)

3. wati (Cam)

1. mấlu (Leh)

2. málu (Pec)

ưál ăža (Leh)

'agua caliente'

ášan-yámba (Leh)

'está caliente'

cólo (Pec)

sisís $(\mathrm{Pec})$

ocón (Pec)

1. k'iN (Cam)

2. k'in (Leh)

kamōti (Leh) 
86. Cangrejo

87. Cantar

88. Cántaro

89. Caña brava

90. Caña de azúcar

91. Carago

92. Carne

93. Carpintero (pájaro)

94. Casa

95. Catalna

96. Catarro

97. Ceiba

98. Ceja

99. Ceniza

100. Cinco

101. Cera

102. Cerrar

103. Cesta

104. Claro

105. Cocodrilo

106. Codo

107. Codorniz jam

yam $(\mathrm{Pec})$

(Campbell registra

kangerehu-na)

ihwa-

juk' in

tukupa

wat

janawt' in

wala

čehe

t'wa

ijuacámba (Pec)

$3^{\text {a }}$. Sg. Pte.

1. ÿúk'in (Leh)

2. iūc'-k'jín (Pec)

(Campbell kantaru-na)

tugú (-ba) (Leh)

1. wat (Cam)

2. ưút (Leh)

3. wat $(\mathrm{Pec})$

ÿắnáưt' in (Leh)

1. waša (Cam)

2. uuấža ưấšấ-na (Leh)

3. wáshia (Pec)

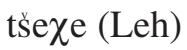

1. t'aw (Cam)

2. t'ấu (Leh)

3. t’au (táu) (Pec)

4. au (Sap)

5. tahu (Alc)

mạ̄lòngấtē (Leh)

(avispón negro)

næê-těn (Leh)

ưat' in (Leh)

pi-zăp-tšúma (Leh)

(lit. 'pelo del ojo)

pilil

1. pílil (Leh)

2. pilíl (Pec)

ts'aj

1. ts'ái (Leh)

2. ts'ai (Pec)

3. tzay (Sap)

yúùa (Leh)

ingapón (Leh)

šígit (Leh)

džămba (Leh)

(véase bueno)

janaw

1. ÿấnau (Leh)

2. yá-nau (Pec)

'lagarto'

kạlnạiNklustu

puri mā-gălna-ínk'ǔštú-na (Leh)

púri (Leh) 


\begin{tabular}{|c|c|c|}
\hline 108. Cola & ts'oh & $\begin{array}{l}\text { 1. i-ts'ó } \chi \text {-na ₹́1-ts'o (Leh) } \\
\text { 2. it-tso (Pec) }\end{array}$ \\
\hline 109. Colocar (se) & rwis- & rūisón (Leh) \\
\hline \multirow[t]{2}{*}{ 110. Comal } & k'elkin & 1. k’èlgin' (Leh) \\
\hline & & $\begin{array}{l}\text { 2. kélgui (Pec) } \\
\text { (Campbell kumal) }\end{array}$ \\
\hline 111. Comején & si & si (Leh) \\
\hline 112. Comer & rom- & rōmón (Leh) \\
\hline 113. Comida & romkin & rōmgin (Leh) \\
\hline \multirow[t]{2}{*}{ 114. ¿Cómo? } & kifa & kīšấ-ba (Leh) \\
\hline & & ¿Cómo es? \\
\hline 115. Comprar & liwa- & ligǔacón $(\mathrm{Pec})$ \\
\hline 116. Con & ten & $\begin{array}{l}\text { mấ-dèn' (Leh) } \\
\text { 'contigo' }\end{array}$ \\
\hline \multirow[t]{2}{*}{ 117. Conga (pez) } & Jirih & 1. shirísna $(\mathrm{Pec})$ \\
\hline & & 2. šíri (Leh) \\
\hline 118. Copinol & palpal & pấlbǎl (Leh) \\
\hline 119. Corazón & imapil & imábil (Leh) \\
\hline 120. Correr & meaji- & meayicón (Pec) \\
\hline 121. Cortar & $t^{\prime} i-$ & i-t'ixión (Leh) \\
\hline \multirow[t]{2}{*}{ 122. Corteza } & ts'sts'a & 1. ts'á-ts'a (Leh) \\
\hline & & 2. ts’á-ts’a (Pec) \\
\hline \multirow[t]{2}{*}{ 123. Coser } & k'or- & k’orámba (Leh) \\
\hline & & $3^{\mathrm{a}}$. Sg. Pte. \\
\hline \multirow[t]{3}{*}{ 124. Cuatro } & sā & 1. sā (Leh) \\
\hline & & 2. sá (Pec) \\
\hline & & 3. sa (Sap) \\
\hline \multirow[t]{2}{*}{ 125. Coyote } & $\int_{w a}$ & 1. šúa (Leh) \\
\hline & & 2. shuás $(\mathrm{Pec})$ \\
\hline 126. Crecer (el río) & atalipo- & 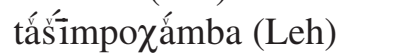 \\
\hline 127. Cresta & c'orc'a & i-chorchá-na (Pec) \\
\hline 128. Criba & kojN & kóin (Leh) \\
\hline 129. ¿Cuánto? & taki & tági (Leh) \\
\hline 130. Cuatro ojos (pez) & sajum & sấ-ÿum (Leh) \\
\hline \multirow[t]{2}{*}{ 131. Cuchara } & waskin & 1. ưáskin (Leh) \\
\hline & & 2. wás-kin (Pec) \\
\hline \multirow[t]{2}{*}{ 132. Cuerno } & $\mathrm{ka} \int \mathrm{u}$ & gáshu (Pec) \\
\hline & & (del cast. 'cacho') \\
\hline \multirow[t]{2}{*}{ 133. Cuita (de pájaro) } & $\operatorname{mam} \int$ oko & mam-žogō-na (Leh) \\
\hline & & (lit. 'excremento blanco') \\
\hline \multirow[t]{2}{*}{ 134. Cumbrera } & t'aw i-oso & t’áu-ihóso (Pec) \\
\hline & & (Lit. 'cabeza de la casa') \\
\hline 135. Cuñada & amte & ámde (Leh) \\
\hline 136. Cuñado & eme & pi-æ̂́me (Leh) \\
\hline
\end{tabular}


137. Cusuco

138. Chancha

139. Chancho

140. Chancho

141. Chaparro

142. Chichicaste

143. Chichiltoto

144. Chilate

145. Chilillo

146. Chinchiguaste

147. Chismuyo

148. Chupar

149. Dar

150. Decir

151. Dedo de la mano

152. índice

153. meñique

154. del pie

155. Defecar

156. Desear

157. Desnudo, estar

158. Día

(cf. sol, calor)

159. Diente

160. Dos

161. Dios

162. ¿Dónde?

163. Dormir

164. Dulce

165. El, ella p\|orp\|or

map'it pere

map'it

map'it kotan

warawal

opopolelele

Jokajka

ola

kajuku

Jepepe

ts'ipolka

ts'u-

mi-

aj-

kofaka kuman

kofaka nepts'ela

kofaka ruku

waJaka kuman

po-

Jej-

ekolo-

jafa

neh

pe

elentios

anuN

pa-

tonka

i-nani
1. p.olp'ol (Cam)

2. púr ruuur (Leh)

3. porpor (Pec)

map'it pere (Cam)

1. map'it (Cam)

2. mấpit ${ }^{n}(\mathrm{Leh})$

3. mapít (Pec)

4. mapit (Alc)

1. map'itkotaN (Cam)

2. mapit ${ }^{\mathrm{n}} \operatorname{kotan}^{-}(\mathrm{Leh})$

3. mapít-cotán (Pec)

uuarăáưaš (Leh)

ỏbốbo-žèllálè (Leh)

šōgaiga (Leh)

ola (Pec)

cayúgu $(\mathrm{Pec})$

1. šx̧ốbè (Leh)

2. shevéve (Pec)

ts'íbólga (Leh)

imu-ts'úga (Leh) 'chupar'

mikón (Leh)

āÿón (Leh)

-gošắga-guman (Leh)

-gošága-něptz'éla (Leh)

gošáka-rúgu (Leh)

ǔašāga-gúmàn (Leh)

pōón (Leh)

-žēỹón (Leh)

x̂kālo-ÿámba (Leh)

yaáza bî́s (Leh)

1. pí-nè, mānês-na

net (Leh)

2. ines-na (Pec)

1. pæe (Leh)

2. be (Pec)

3. pé (Sap)

pi-elén Dios (Leh)

('nuestro tatita Dios')

ánun (Leh)

pakón (Leh)

1. toNga (-na) (Cam)

2. tón'ga (Leh)

3. tónga (Pec)

i-nắni (Leh) 
166. Ellos (as)

167. Empezar

168. En

169. Enagua

170. Encinta, estar

(cf. vientre, panza)

171. Enfermo, estar

172. Enterrar

173. Entrar

174. Escoba

175. Escorpión (reptil)

176. Escorpión (artrópodo)

177. Esperar

178. Espina de pescado

179. Esposa (cf. mujer)

180. Estar de pie

181. Estómago

182. Estornudar

183. Estrella

184. Excrementos

(cf. cuita de pájaro)

185. Extinguir

186. Familia

187. Fiebre

188. Filín (pez)

189. Flaco

190. Flor

191. Fluir

a-nani
ifipe-
k'ati
lamakin
k'omo-anpa
ona-
lat'u-
apta-
t'apkin
arankaka
sojojo
Ver aguardar
ma
sija

pais- pas-

ints'iliwakah kofujim-

sirih

mam

muj-

hwamilja

jaja

kojom

konko

Jila

mjasa- ā-năni (Leh)

íšipeášba (Leh)

$3^{a}$. sg. Perfecto

uuálk'ádi (Leh)

('en el agua')

1. lāmắgin (Leh)

2. lamágui (Pec)

k'ómō-ámba (Leh)

ōnakón (Leh)

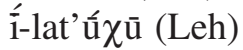

'lo enterré'

aptacón (Pec)

'voy a entrar'

1. t'aṕkin' (Leh)

2. t'ap-kin (Pec)

ára'ngấka (Leh)

sơyóöo

mấ-na (Leh)

1. siya (Cam)

2. sî̀a rí́a (Leh)

3. í'dsia (Pec)

paisón (Leh)

'estoy parado'

păsámba 'está parado'

ints'iliwagah (Cam)

kǒžužimámba (Leh)

$3^{\text {a }}$. sg. pte.

1. síri siríx-na (Leh)

2. círi (Pec)

màm-ba (Leh)

imu ÿámba (Leh)

u-hwamilya-na (Cam)

ÿấža (Leh)

1. kóyoN (Cam)

2. coyóm $(\mathrm{Pec})$

3. kô-ÿùm (Leh)

1. kòn'gō (Leh)

2. cóngo $(\mathrm{Pec})$

1. šila (Leh)

2. shíla (Pec)

mīāsa-ōkámba (Leh)

$3^{\text {a }}$. sg. Futuro 


\begin{tabular}{|c|c|c|}
\hline 192. Frente & tits'e & $\begin{array}{l}\text { 1. pi-dits'e (Leh) } \\
\text { 2. idi-tsé (Pec) }\end{array}$ \\
\hline \multirow[t]{4}{*}{ 193. Frijol } & \multirow[t]{4}{*}{ Juli } & 1. šuli (Cam) \\
\hline & & 2. šúli (Leh) \\
\hline & & 3. shuli (Pec) \\
\hline & & 4. xúli (Sap) \\
\hline \multirow[t]{3}{*}{ 194. Frío } & \multirow[t]{3}{*}{ ts'ana } & 1. in-ts'ana (Cam) \\
\hline & & 2. ts’án'gažá (ba) (Leh) \\
\hline & & 3. ts’ána (Pec) \\
\hline \multirow[t]{2}{*}{ 195. Fruto } & \multirow[t]{2}{*}{ Jea } & 1. séa $(\mathrm{Pec})$ \\
\hline & & 2. žéa (Leh) \\
\hline \multirow[t]{4}{*}{ 196. Fuego } & \multirow[t]{4}{*}{ ik'an } & 1. ik'an ik'aN (Cam) \\
\hline & & 2. ík’àn’ (Leh) \\
\hline & & 3. ic'an (pec) \\
\hline & & 4. ícang (Sap) \\
\hline \multirow[t]{2}{*}{ 197. Fuera ( al exterior) } & \multirow[t]{2}{*}{ jota } & ú-t’áu-ÿốta (Leh) \\
\hline & & 'fuera de mi casa' \\
\hline 198. Fumar & ofo- & ōžokón (Leh) \\
\hline \multirow[t]{4}{*}{ 199. Gallina } & \multirow[t]{4}{*}{ kaflan } & 1. kašlaN (Cam) \\
\hline & & 2. kášlan` (Leh) \\
\hline & & 3. cashlan (Pec) \\
\hline & & 4. caishlan (Alc) \\
\hline \multirow[t]{2}{*}{ 200. ___ de monte } & \multirow[t]{2}{*}{ kotan ka lan } & 1. kốtàn'-kášlan’ (Leh) \\
\hline & & 2. cotán-cashlan (Pec) \\
\hline 201. Gallo & ka〕lan-kew & kášlan'-kêū (Leh) \\
\hline 202. Garganta & ts'uru & ts'úru (Leh) \\
\hline 203. Garza blanca & walwatu〕 & ǔǎl-ǔádūš (Leh) \\
\hline 204.Garzón & oho & ắxo (Leh) \\
\hline \multirow[t]{2}{*}{ 205. Garrobo } & \multirow[t]{2}{*}{ mer } & 1. mérn (Leh) \\
\hline & & 2. $\operatorname{mer}(\mathrm{Pec})$ \\
\hline \multirow[t]{3}{*}{ 206. Gato } & \multirow[t]{3}{*}{ mitsu } & 1. mistu (Cam) \\
\hline & & $\begin{array}{l}\text { 2. místu, (Leh) místo, } \\
\text { míšto }\end{array}$ \\
\hline & & 3. mishto (Alc) \\
\hline \multirow[t]{2}{*}{ 207. ___ de monte } & \multirow[t]{2}{*}{ kotN mistu } & 1. kốtàn'-místu (Leh) \\
\hline & & 2. cotán-mistú (Pec) \\
\hline \multirow[t]{3}{*}{ 208. Gavilán } & \multirow[t]{3}{*}{ muk'ika } & 1. muk'iga (Cam) \\
\hline & & 2. mūîga (Leh) \\
\hline & & 3. muquiga (Pec) \\
\hline \multirow[t]{2}{*}{ 209. Golpear (tambor) } & \multirow[t]{2}{*}{ mal- } & māžámba (Leh) \\
\hline & & $3^{\mathrm{a}} . \mathrm{sg} \cdot \mathrm{pte}$ \\
\hline 210. Gordo & pola & bốla (Leh) \\
\hline 211. Gotera & kurara & kuřera (Cam) \\
\hline
\end{tabular}


212. Grande

213. Grano

214.Guabo

215. Guacal

216. Guapote (pez)

217.Guaruno (palo de )

218. Guasalo (tucán)

219. Guatusa

220. Guayabo

221. Guineo

222. Gusano

223. Hablar

224. Hacer

225. Hacha (de piedra)

226. Hamaca

227. Herir

228. Hermana mayor

229. Hermana menor

230. Hermano mayor

231.Hermano menor

232. Hígado

233. Hija

234.Hijo (a)

235. Hilo

236. Hinchar (se)

237. Hoja

238. Hombre

239. Hombro

240. Hormiga

$$
\begin{aligned}
& \text { puka } \\
& \text { i } \int a m a n \\
& \text { ver fruto } \\
& \text { pater }
\end{aligned}
$$

k'ama

polul

ts'awa'sum

ts'ewe

keke

tororo

inkinja

ts'its'a

Jakini-

ji-

patkin

saw

t'oj-

peleh

c'ah

koh

Ver Hermana (menor)

muts'u

jusija púga (Leh)

išamán (Cam)

pấdēr (Leh)

1. k’áma (Cam)

2. k’áma (Leh)

pấlùl (Leh)

ts'áừa-zún (Leh)

ts' æ̂̀ứx-na (Leh)

kæékē (Leh)

torốro (Leh)

in'gínia (Leh)

ts'its'a (Leh)

šaginikón (Leh)

šikón (Leh)

pấtkin' (Leh)

1. sāu t'u (Leh)

2. sáug'-do (Pec)

t'óaỹámba, áraÿámba

$3^{\mathrm{a}} \mathrm{sg}$. Pte

ū-bæêlấ $\chi$-na (Leh)

ū-ts'á $\chi$-na (Leh)

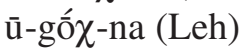

mūts'ú-na (Leh)

1. yu-siya (Cam) 'hija'

2. ū-ÿú-sián-nna (Leh) 'hija'

(ju 'hijo'; sija 'mujer')

3. ù-ÿú-na (Camb) 'hijo'

4. ū-ÿúu-na (Leh) 'hijo' máyu (Pec) 'hijo'

ju

rif

rats'sa-

aw

ifko

mun

wank'ama

its'its'i rìs (Leh)

rấts'a ÿámba (Leh)

'está hinchado'

1. âu (Leh)

2. iyau (Pec)

1. iško (Cam)

2. íškō (Leh)

3. íšhco (Pec)

4. íxco (Sap)

mún $(\mathrm{Pec})$

ủank'ấma (Leh)

its'íts'i (Leh) 
241. Hoy

242. Hueso

243. Huevo

244. Huipil

245. Humo

246. Iguana

247. Ilama (pez)

248. Intestinos

249. Ir

250. Jaguar

251. Jefe de la casa

252. Jícaro (palo de)

253. Jicote

254. Jiote (árbol)

255. Jocote amarillo (dulce)

256. garrobero

257. Jolote (chompipe)

258. Joven (cf. hijo)

259. Jugar

260. Ladino

261. Lagartija

262. Lágrima

263. Lapa

264. Largo

265. Laurel

266. Lavar (se)

267. Lejos (Cf. Largo)

268. Lengua

269. Lengüeta del telar

270. Leña

271. Levantarse

272. Limpio

273. Luna

274. (eclipse de ) pitu

fila-ts'e

kua

leke

mo

mer-tsojkan

ilama

manfuli

o-

lepa

matarim

k'ama suN

whele japilko

sotts'ats'a

muraka

muraka-ts'a i

lolo

ju

inkini-

moso

kofolka

kaju

ewa

kota

Juman

ts'i-

nepal

tje-nepal

fah

pas-

fam

lets'a

lets'a kalikanpa pídu (Leh)

šíla-ts'é (Leh)

1. kúa (Leh)

2. cúa (Pec)

lốkè (Leh)

mố-na (Leh)

mèrn-ts'ōíkản (Leh)

ilấma (Leh)

1. mā-mănšúli (Leh)

2. imanshúli (Pec)

ōkón' (Leh)

léba (Leh)

matárim (Pec)

k'ấma-žún' (Leh; la ž

parece errata por $\mathrm{z}$ )

uésle-ȳāpíškō (Leh)

sot'tsátsa (Pec)

mūrāka (Leh)

múrakā-ts'ári (Leh)

lôlo (Leh)

ÿú-na (Leh)

ínginikámba (Leh)

$3^{\mathrm{a}} \mathrm{sg}$. Pte.

mốzō-ísko (Leh) 'ladino'

kōžólga (Leh)

kấ̈u (Leh)

x̂́u (Leh)

katấ-ba (Leh)

šúmàn (Leh)

ts'íxón (Leh)

nēbăl-na (Leh)

tíè-nếbăl (Leh)

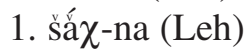

2. ša (Cam)

3. sha (Pec)

4. ra (Sap)

pasón (Leh)

džấmba (Leh; véase

'bueno')

1. lêts'à (Leh)

2. lésa-na (Pec)

3. létzaj (Sap)

lếts’à kấli kámba (Leh)

(lit. 'la luna está enferma') 
275. nueva

276. Luz

277. Llama

278. Llamar

279. Llanura, llano

280. Llorar

281. Llorón

282. Llover

283. Llovizna

284. Lluvia

285. Madre

286. Maduro

287. Maguey

288. Marcillo

289. Maíz

290.

291. camagua

292. cocido (cf. niztamal)

293. conducir el

294. desgranar el

295. destusar el grano de

296. harina de (Cf. Pinol)

297. negrito

298. pelo de (Cf. Cabello)

299. tusa de

300. Malacate

301. Mandíbula superior

302. Mango (de hacha) (cf. mano) 303. Mano

304. Mañana (parte del día) lets'a mali

wesa

ik'an-e-nepal

mawa-

lak'a

kaju-

kaits'ulu

Jojikanpa

Jomi $\int_{a N}$

$\int_{\mathrm{o}}$

lanke

Jela

wasata

imats'ili

ima

imats'as

talkin sea

ukuji-

ima um-

ima tipa-

ima seja

ima p'uru」

ima puri

imalah

p'orof

ri $\int \mathrm{kiN}_{\mathrm{N}}$

kamats'e

kofaka

ko〕aka

wele lếts'à mấli (Leh)

1. ủēsấ-na (Leh)

2. wse-ana (Pec)

ík'àn-x̂̀-nếbăl (Leh)

(lit. 'lengua de fuego')

mawajión (Pec)

lăk'a (Leh)

cayucón cayocón (Pec)

kaits'úlu (Leh)

šǒ-žikámba (Leh)

'está lloviendo'

1. šò-míš́ăn' (Leh)

2. shoomishmish (Pec)

š́ (Leh)

1. ū-lắn'gè (Leh)

2. ilangui (Pec)

šéla (Leh)

wasadá $(\mathrm{Pec})$

ímats'hili (Pec)

1. ima (Cam)

2. íma (Leh)

3. íma (Pec)

4. íma (Sap)

imấ-ts'às (Leh)

talgin-dséa (Pec)

ima-ugushicón (Pec)

íma-umámba (Pec)

ímatipár-kin (Pec)

1. ima-zéȳa (Leh)

2. ima-dséa (Pec)

íma púruš (Leh)

íma-buri (Pec)

1. imála (Leh)

2. íma-alásh $(\mathrm{Pec})$

1. hoórǒš (Leh)

2. porosh $(\mathrm{Pec})$

ríš̀-kin (Leh)

pì-gamấ-tz'è (Leh)

gošánga (Leh)

1. košaka (Cam)

2. pí-gờắga (Leh)

3. igoshagá-na (Pec)

4. coxága (Sap)

gứelè (Leh) 


\begin{tabular}{|c|c|c|}
\hline \multirow[t]{3}{*}{ 305.Mapachín } & \multirow[t]{3}{*}{ wala } & 1. wala (Cam) \\
\hline & & 2. ưála (Leh) \\
\hline & & 3. wala (Pec) \\
\hline 306. Mar & saj & sai $($ Leh $)$ \\
\hline \multirow[t]{3}{*}{ 307. Marido } & jiku & 1. u-ÿigú́-na (Leh) \\
\hline & & 'el marido mío' \\
\hline & & 2. máiu (Pec); mạ ‘tu' \\
\hline 308. Masticar & ro- & rōmámba (Leh) \\
\hline 309. Matar & Jil- & -žilón (Leh) \\
\hline 310. Matate (red) & sau & sấu (Leh) \\
\hline \multirow[t]{2}{*}{ 311. Mear } & ila- & ilakámba (Leh) \\
\hline & & $3^{\mathrm{a}} \mathrm{sg}$. Pte. \\
\hline 312. Mecapal & mi $\int$ t' eaka & mišt'ēeága (Leh) \\
\hline \multirow[t]{2}{*}{ 313. Medianoche } & welataku & 1. uvéla-dáku (Leh) \\
\hline & & 2. welá-ra (Pec) \\
\hline 314. Medio & taku & dǎkú-na (Leh) \\
\hline 315. Mediodía & jaltaku & ÿáz-takù-áška (Leh) 'es \\
\hline & & $\begin{array}{l}\text { mediodía' (la } \underline{z} \text { es error } \\
\text { por ž) }\end{array}$ \\
\hline 316. Menguar (la luna) & kali- & kálikámba (Leh) \\
\hline 317. Mezcal & wa $\int \mathrm{a}$ & uấža (Leh) \\
\hline \multirow[t]{3}{*}{ 318. Miel } & wehle & 1. wehle (Cam) \\
\hline & & 2. ưéslè (Leh) \\
\hline & & 3. wesle (Pec) \\
\hline \multirow[t]{3}{*}{ 319. Milpa } & ta & 1. ta- $(\mathrm{Cam})$ \\
\hline & & 2. ta (Leh) \\
\hline & & 3. tána $(\mathrm{Pec})$ \\
\hline 320. desyerbar la & muntawa- & mundahuacón (Pec) \\
\hline 321. doblar la & kut- & cutámba $(\mathrm{Pec})$ \\
\hline 322. $\overline{\text { Mirar }}$ & č'i- & tš’ikón (Leh) \\
\hline 323. Mitad & taku & Cf. medio \\
\hline 324. Mojar & ts'i- & Cf. lavar \\
\hline 325. Moler & maali- & mâấ likớn (Leh) \\
\hline 326. Monda Metálica & tumi & tumí-na (Leh) \\
\hline \multirow[t]{2}{*}{ 327. Montaña } & kotan & 1. kotaN (Cam) \\
\hline & & 2. kốtăn` (Leh) \\
\hline \multirow[t]{2}{*}{ 328. Morder } & ti- & idímba (Leh) \\
\hline & & $3^{\mathrm{a}} \mathrm{sg}$. Pte. \\
\hline 329. Morir & Sil- & šilón (Leh) \\
\hline 330. Mosca & ts'irifir & tsirížì (Leh) \\
\hline \multirow[t]{2}{*}{ 331. Mover (se) } & lim- & lūmámba (Leh) \\
\hline & & $3^{\mathrm{a}} \mathrm{sg}$. Pte. \\
\hline 332. Muchacha & sija-ju-wewe & sīa-ÿūu-uécuēē (Leh) \\
\hline 333. Mucho & johan & ÿóxăn (Leh) \\
\hline 334.Muerto & fila & šila (Leh) \\
\hline
\end{tabular}


335. Mujer

336. Murciélago

337. Músculo del brazo (bíceps)

338. Muslo (Cf. Pierna)

339. Nacer

340. Nalgas

341. Nance

342. Nariz

343.Negro

344. Nido

345. Niebla

346. Nieto

347. Nigua

348. Niño

349. Niztamal (cf. maíz cocido)

350. noche

351. Nosotros

352. Nube

353. Nuca

354. Nudo.

355. Nuera

356. Nuevo

357. Nato

358. Ocote

359. Oír

360. Ojo sija

1. siya (Cam)

2. síỹa sía (Leh)

3. í'dsia (Pec)

4. silla (Alc)

5. síya (Sap)

mits'on

1. míts'òn (Leh)

2. mishon (Alc)

kalkatu

poh-puka

tul-

miri

t'alna

nepkuru

siska

mā-gál-gatú-na (Leh)

pī-bōs-pugấ-na (Leh)

tūlón (Leh)

mā-mirí-na (Leh)

t’álna (Leh)

1. pi-něpkurú-na (Leh)

2. nép-kúru (Pec)

1. sínga (Leh)

2. sígna $(\mathrm{Pec})$

ja

Ver Nube

latan

map'it-t'ut'u

iăš (Leh)

ū-ladắn`-na (Leh) 'nieto'

u-ladán'-na-síÿa 'nieta'

māpit-t'ūt'u (Leh)

(lit. 'pulga gorda')

(map'it 'chancho')

wewe, ju wewe

1. yu-wewe (Cam)

2. uvéùè (Leh); ÿu-ưéu้e

3. wíwí (Pec)

4. yuéwe (Sap)

tấlgin sía (Leh)

tálgin žéa (Leh)

ts'upa

ts'ūb-ấšba (Leh)

('ya está oscuro')

pi-nani pi-nấni (Leh)

ts'upa 1. ts'upa- (Cam)

2. ts'úba'ts'úba (Leh)

$\begin{array}{ll}\text { t'ant'an } & \text { t'ant'án-na (Leh) } \\ \text { kukura } & \text { kūgúra (Leh) }\end{array}$

amte sija ù-amdé-na-síÿa (Leh)

tuja

nepkuru leka $\int_{a}$

fal

en (i)

dúÿa (Leh)

nēpkúru-lēgấša (Leh)

šāl (Leh)

i-ếnikámba (Leh)

$3^{\mathrm{a}}$. sg. Pte.

1. u-sat-na (Cam)

2. pi-zấp-na(Leh)

3. dsáp (Pec) 


\begin{tabular}{|c|c|c|}
\hline 361. __ de agua & wal i sap & ủăl-ī-zăp (Leh) \\
\hline 362. $\overline{\text { Oler }}$ & $\int u \int i_{-}$ & i-žu-šikón’ (Leh) \\
\hline \multirow[t]{2}{*}{ 363. Olomina } & orum & 1. ốrùm (Leh) \\
\hline & & 2. orómna (Pec) \\
\hline \multirow[t]{2}{*}{ 364. Olote } & ima-toh & 1. imấ-tă $\chi$ (Leh) \\
\hline & & 2. ema-to (Pec) \\
\hline 365. Ombligo & k’ul & kúl-na (leh) \\
\hline \multirow[t]{3}{*}{ 366. Oreja } & tokoro & 1. tokoro (Cam) \\
\hline & & 2. pi-dogốro (Leh) \\
\hline & & 3. idsogoró-na (Pec) \\
\hline 367. Orina & ila & íla- (Leh) \\
\hline 368. Oro & oropa & orố-ba (Leh) \\
\hline \multirow[t]{3}{*}{ 369. Oscuro, estar } & ts'upa & 1. ts'úba-ká́mba (Leh) \\
\hline & & 2. sup-áshpa (Pec) \\
\hline & & 3. uc'uwaba (Cam) \\
\hline \multirow[t]{2}{*}{ 370. Pachón } & ts'ui & tsits’a-ts’úi (Leh) \\
\hline & & 'gusano pachón' \\
\hline \multirow[t]{2}{*}{ 371. Padre } & t'ani & 1. $\overline{\mathrm{u}}-\mathrm{t}^{\prime} a \hat{n} \mathrm{e}^{\sim} \overline{\mathrm{u}}-\mathrm{t}^{\prime} a \hat{n} \mathrm{i}(\mathrm{Leh})$ \\
\hline & & 2. itani $(\mathrm{Pec})$ \\
\hline 372. Paja & $\int \mathrm{a} \int \mathrm{a}$ & shásha (Pec) \\
\hline 373. Pájaro & ts'imts'im & ts'ímts'im (Leh) \\
\hline \multirow[t]{2}{*}{ 374. Palma de coyol } & juki & 1. yugu (Cam) \\
\hline & & 2. ÿúgu (Leh) \\
\hline \multirow[t]{2}{*}{ 375. Paloma de San Nicolás } & t'urup & 1. t'úrup (Leh) \\
\hline & & 2. turúp $(\mathrm{Pec})$ \\
\hline 376. Pantano & wal ets'a & ǔàl-éts’a (Leh) \\
\hline 377. Paral & set'uaka & cé-th’-hága (Pec) \\
\hline 378. Pedernal & ketam & kæé-dam (Leh) \\
\hline \multirow[t]{3}{*}{ 379. Pared } & Jiwin & 1. šíüin (Leh) \\
\hline & & 2. shiwín (Pec) \\
\hline & & 'barro' \\
\hline \multirow[t]{2}{*}{ 380. Párpado } & sap-ts'ats'a & pi-zap-ts'ấts'à (Leh) \\
\hline & & (lit. 'piel del ojo) \\
\hline 381. Pasado mañana & peni & pæ̂́-ni (Leh) \\
\hline (en dos días) & & \\
\hline \multirow[t]{3}{*}{ 382. Pato } & patul & 1. pấduš (Leh) \\
\hline & & 2. wal-badush (Pec) \\
\hline & & 3. patu (na) (Cam) \\
\hline 383. Pava & kuikui & kúigùi (Leh) \\
\hline \multirow[t]{2}{*}{ 384. Peine } & tenmaskin & ten`-má́skin (Leh) \\
\hline & & (¿mal 'golpear’?) \\
\hline $\begin{array}{l}\text { 385. Pelo de animal } \\
\text { (cf. cabello) }\end{array}$ & Jumah & i-žūma i-šúma (Leh) \\
\hline 386. Pene & t'em & t'ém tèn`-na (Leh) \\
\hline \multirow{2}{*}{ 387. Pequeño } & ts'iri ${ }^{\prime} \mathrm{ki}$ & ts'íriškí-ba (Leh) \\
\hline & wewe & u้éu ē \\
\hline
\end{tabular}


388. Perilla

389. Periquito

390. Perro

391. Pesado

392. Petate

393. Pez

394. verde

395. picado

396. Picar

397. Pico

398. Pie

399. Piedra

400. de afilar

401. de moler maíz

(metate)

402. mano de

403. Piel

404. Pierna

405. Pimienta (cf. aguacate)

406. Pino

407. Pinol (pinolillo)

408. Piña

409. Piñuela cimarrona

410. Piojo

411. Pita

412. Pito (árbol)

413. Plátano

414. maduro

415. verde
iN $\int u m a h$

wirih

$\int \mathrm{u} \int \mathrm{u}$

t'ili

apan

Jok' in

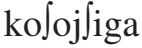

tulu

ti

ints'eh

wa〕aka

ke

kali

mohmon

mohmonko〕aka

ts'ats'a

poh

Jik'a

Jalk'i

p'urus

mats'ati

muraka tsari

tem

ka

t'uku

pula $\int$

pula $\int$ Jela

pule $\int$ ohan in-džúma (Leh)

1. wírih (Cam)

2. ứri (Leh)

3. wirí (Pec)

1. šušu šušu (Cam)

2. šúžu-šúrūū (Leh)

3. shúshu (Pec)

t'ili-ba (Leh)

1. âbăn (Leh)

2. ában $(\mathrm{Pec})$

1. šok'iN $\sim$ so ? iN (Cam)

2. šókin (Leh)

3. shókin (Pec)

košoyšiga (Cam)

íma-túlu (Pec)

'maíz picado' idímba 'le picó'

in-ts' æé $\chi$-na

1. pi-ủašấga (Leh)

2. washagá-na (Pec)

kæ̂́ (Leh)

kážì (Leh)

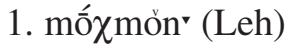

2. mooj-shon (Pec)

3. molmo (Sap)

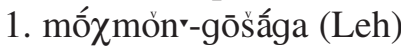

2. móoj-goshága (Pec)

1. ts'ấts'a (Leh)

2. itsá-tsa (Pec)

1. pī-bōs-na (Leh)

2. ibó-na (Pec)

šík'à (Leh)

shal'i (Pec)

p'urus (Leh)

mats'ấdi (Leh)

múraka-ts'ári (Leh)

1. tem (Cam)

2. tấm (Leh)

ká (Pec)

t'úgu (Leh)

1. púlǎs (Leh)

2. pulásh (Pec)

(Prést.)

púlàš šếla (Leh)

púlàš-ốsăn (Leh) 
416. Pléyades

(Siete Cabritas)

417. Pluma

418. Poco

419. Podrir (se)

420. Polvo

421. Pollo

422. Poner

423. Ponerse (el sol)

424. ¿Por qué?

425. Poste (horón)

426. hoyo del

427. Poza

428. Pozol

429. Pueblo

430. Puerta

431. Pues

432. Quebrada

433. Pulga (Ver Nigua)

434. Pulido

435. Pupila

436. Pus

437. ¿Qué?

438. Quebracho

439. Quebrar

440. Quedarse

441. Quemar

442. Quemazón

443. Querque (vultúrido)

444. ¿Quién?

445. Quijongo

446. Quiquisque

447. Rana ts'ujlawa

Juma

č'inkira

lukawa-

tula

kaslaNwewe

ruis-

ata pe-

kaspa aj a

watara

ora

ojoj

mulukwa

ek'e

ink' oran

tala

wara-ju

t'ut'u

potan

sapsea

impama

kas

silisu

pol-

mea-

oporči-

k'ulila

kerker

k'ula

karanpa

kohaw

kopopo-ts'oikan
1. ts'úi-láủa (Leh)

2. t'tsú-iláwa (Pec)

šúma (Leh)

tšin'gíra (Leh)

lūkấüa-kámba (Leh)

$3^{\mathrm{a}}$ sg. Pte.

túla (Leh)

kašlań-ưếūē (Leh)

cashlan-güvi (Alc)

rūisón (Leh)

$1^{\mathrm{a}}$ sg. Pte.

ỹâža áta-pæấása (Leh)

'el sol se puso'

kăs-pá aîža (Leh)

1. watařa (Cam)

2. ủatấra (Leh)

3. huatára $(\mathrm{Pec})$

óra (Pec)

oioy (Pec)

mús s̄u gúa (Leh)

1. æấ k'è (Leh)

2. e?e (Cam)

1. in-k'ốran (Leh)

2. lé-íncóran (Pec)

dála (Pec)

1. u้arr-i-̈̈u (Leh)

2. warrá-yu (Pec)

3. guarra-yu (Alc)

t'úto (Leh)

pốtan, (Leh)

pí-zàp-séa (Leh)

(lit. 'semilla del ojo')

imbắma (Leh)

kàs (Leh)

síli-sūn (Leh)

pólkažăma (Leh)

'quebrado'

mēakón` (Leh)

obǒrtšikámba (Leh)

k'ū líla (Leh)

quérquer (Pec)

k'úla (Leh)

karámba (Leh) (Prést.)

kóxâu (Leh)

kobốbo ts'ōikan (Leh) 
448. Rápido

449. Raspaguacal (ver Chaparro)

450. Rata

451. Ratón

452. Reír

453. Remedio

454. Respirar

455. Reumatismo

456. Río

457. Roble

458. Rojo

459. Romper

460. Ropa

461. Rostro

462. Rozar

463. Sábana

464. Saber

465. Saíno

466. Sal

467. Seis

468. Salamanqués

(especie de lagartija)

469. Salir

470. Saliva

471. Sangre mapala

warawal

k'uwa

k'uwaruri

jolo-

liwena

ink'ala-

ujika

wara

malsum

wesan

erc'i-

lamtara

dits'e (Ver Frente)

tapu-

laliup-kiN

ti-

Ver Chancho de monte ts'epe

wi

kariki

ata-

inwal

ala mabala (Cam)

ưárāuuáš-na (Leh)

k'úùa (Leh)

1. k'uwa (Cam)

2. k'úa-rúri (Leh)

3. cúa (Pec)

ȳōlokón (Leh)

í-liừína (Leh)

ink'ala kắmba (Leh)

$3^{\mathrm{a}} \mathrm{sg}$. pte

užíga (Leh)

1. war̄a (Cam)

2. uuárra (Leh)

3. warrá (Pec)

4. guarra (Alc)

măl-sún (Leh)

1. ưéšan (Leh)

2. wísan (Pec)

iertš'ikón' (Leh)

'lo rompo'

1. làmpt’ấra

lămpấra (Leh)

2. lamdará (Pec)

pi-díts'è (Leh)

'nuestro rostro'

tab

lashiúp'kin (Pec)

ídikăn`-gé-u (Leh)

'no lo sé'

1. ts'epe (Cam)

2. ts'æâbē (Leh)

1. ưí (Leh)

2. wí (Pec)

3. ví (Sap)

karígi (Leh)

ātakón (Leh)

in'-ǔál înd-úăl (Leh)

('lit. 'agua de la boca')

1. ala (Cam)

2. ála (Leh) 


\begin{tabular}{|c|c|c|}
\hline 472. Sapo & k’opopo & $\begin{array}{l}\text { 1. k’obobo }(\mathrm{Cam}) \\
\text { 2. kōbốbo }(\mathrm{Leh}) \\
\text { 3. cobobó (Pec) }\end{array}$ \\
\hline 473. Saradito & kumaru & kumáru (Cam) \\
\hline 474. Sardina & Jaja & šăýy (Leh) \\
\hline 475. Seco & tina & $\begin{array}{l}\text { 1. tínasáába (Leh) } \\
\text { 2. tinába (Pec) }\end{array}$ \\
\hline 476. Sembrar & isa- & isakón (Leh) \\
\hline 477. Semilla & Jea & $\begin{array}{l}\text { 1. sea (Pec) } \\
\text { 2. žéa (Leh) }\end{array}$ \\
\hline 478. Sentarse & rwis- (Ver Colocarse) & rūisón (Leh) \\
\hline 479. Serpiente & amap & $\begin{array}{l}\text { 1. ámàp (Leh) } \\
\text { 2. amap }(\mathrm{Pec})\end{array}$ \\
\hline 480. cascabel & amap sejka & ámap séiga (Leh) \\
\hline 481. masacuata & amap lepa & ámap léba (Leh) \\
\hline 482. ___ zumbadora & amap ts'oikan & ámap ts'oíkan (Leh) \\
\hline 483. Sobrina & anke & $\begin{array}{l}\text { 1. pî̉-aán'gè }(\mathrm{Leh}) \\
\text { 2. maránggie }(\mathrm{Pec})\end{array}$ \\
\hline 484. Sol & $\mathrm{ja} \int_{\mathrm{a}}$ & $\begin{array}{l}\text { 1. yaša (Cam) } \\
\text { 2. ÿăsa (Leh) } \\
\text { 3. iásha (Pec) } \\
\text { 4. yasha (Sap) }\end{array}$ \\
\hline 485. Sombra & sapa & sába (Leh) \\
\hline 486. Sombrero & suat & $\begin{array}{l}\text { 1. súát }(\mathrm{Leh}) \\
\text { 2. súat }(\mathrm{Pec})\end{array}$ \\
\hline 487. Sonar & sima- & $\begin{array}{l}\text { símakámba (Leh) } \\
3^{\text {a }} \text { sg. Pte. }\end{array}$ \\
\hline 488. Soñar & pat'iwa- & pá-t'iưakón' (Leh) \\
\hline 489. Sopa de Carne & walawal & huarsháhual (Pec) \\
\hline 490. Sucio & ulan & úlăn (Leh) \\
\hline 491.Sudar & lawel- & laǔelón (Leh) \\
\hline 492.Sudor & lawela & laù éla (Leh) \\
\hline 493. Suegra & amtesija & $\begin{array}{l}\text { 1. pí-amdèzía (Leh) } \\
\text { 2. maanclesiá (Pec) }\end{array}$ \\
\hline 494. Suegro & amteós & $\begin{array}{l}\text { 1. ămdēốs (Leh) } \\
\text { 2. maamdiós (Pec) }\end{array}$ \\
\hline 495. Supurar & inpam- & ímbamón (Leh) \\
\hline 496. Tabaco & jowa & $\begin{array}{l}\text { ÿốúa (Leh) } \\
\text { lấle (Leh) }\end{array}$ \\
\hline $\begin{array}{l}\text { 497. Tabanco } \\
\text { 498. Tacuacín }\end{array}$ & $\begin{array}{l}\text { lale } \\
\text { ts'ewe }\end{array}$ & $\begin{array}{l}\text { lale (Len) } \\
\text { ts'æ̂́úe (Leh) }\end{array}$ \\
\hline 499. Tal vez & wis & wis (Leh) \\
\hline 500. Talar & wis-ta- & $\begin{array}{l}\text { wístāokokón' (Leh) } \\
1^{\text {a }} \text { sg. futuro }\end{array}$ \\
\hline $\begin{array}{l}\text { 501. Talpetate } \\
\text { (piedra blanca) }\end{array}$ & ke $\int$ oko & kxê-žógo (Leh) \\
\hline
\end{tabular}


502. Tamal

503. También

504. Taparrabo

505. Tapesco

506. Tapir

507.Tapiscar

508. Tarde (hora del día)

509. Temer

510. Temporal (estado del clima)

511. Tarro

512. Tasacual (techo de paja)

513. Techo de la casa

514. Telar

515. Temporal

516. Tener

517. hambre (cf. Morir)

518. sed

519. Terremoto

520. Testículos (los coyoles)

521. Teta (Ccf. chupar)

522. Tía

(Hna de padre)

523. Tierra

524. Tío

(Hno de padre) ni

eslen

sohwara

leh

era-kajo

para-

mari-alpa

sanawa-

Jomon

jikal

tara

t'aw munkola

wik'in

č'uts'a

kaj-

ofil-

waljil-

omon lumanpa

juku

ts'ukin

Ver Hna Mayor

OMON

1. omoN (Cam)

2. ắmün (Leh)

3. homun (Alc)

1. ni (Cam)

2. nì (Leh)

3. ni'-i'-íts-as (Pec)

eslen (Pec)

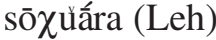

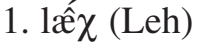

2. lé (Pec)

éra-gáÿú (Leh)

káÿo

ema paracón (Pec)

'arrancar el maíz de las plantas'

mấri-ấšpa (Leh)

sănáủakámba (Leh)

$3^{\mathrm{a}} \mathrm{sg}$. Pte.

šắmờn

1. šigàl (Leh)

2. shiigal (Pec)

(Pipil sikal)

tấra $(\mathrm{Pec})$

t'âuù-mün'góla (Leh)

úik'in (Leh)

1. tš'úts'a (Leh)

2. tshutsha Pec)

u-t'áü-gaÿón (Leh)

'tengo casa'

ǒžilón' (Leh)

gưăl-žil-ón (Leh)

ắmùn lū mámba (Leh)

(lit. 'la tierra se mueve')

má-ÿugú-na (Leh)

1. ts'ūgín'n-na (Leh)

2. it'tsúgui (Pec)

Ver Hno Mayor 
525. Tirar

526. Tizate

527. Tocar

528. Todo

529. Tomate

530. Torcer

531. Tormenta

532. Torogós

533. Tortilla

534. Tortolita

535. Tortuga de río

536. Tos

537. Toser

538. Tostado

539. Tostar

540. Trabajar

541. Traer

542. Transpirar

543. Trasero

544. Trenza

545. Trenzar

546. Tres

547. días

548. Tripas

549. Tú

550. Triste

551. Troj

552. Tronar

el-fi
paji
ete-
inpoajanpa
tomati
ka-
Ver Lluvia
ts'ots'uru
tete

Ver Paloma de

San Martín

t'ef

kolo-

kela

kel-

teket-

ko-

Ver Sudar

Ver Nalgas

mada

mata-

lawa

lawa-ni

Ver intestino

ma-nani

Jilanjanpa

troja

Jo-Jima- ièl-žikămba (Leh)

$3^{a}$ sg. Pte.

pấżi (Leh)

ima-páshi (Pec)

'maíz tizate'

iedejión (Pec)

ímpōaÿámba (Leh)

tomắti (Leh)

(Prést.)

ka-cajión (Pec)

'torcer pita'

ts'óts'úrū (Leh)

1. tete $(\mathrm{Cam})$

2. tấtè (Leh)

3. tété $(\mathrm{Pec})$

t'æ̂̋š (Leh)

kóžǒ (Leh)

kóžo-gámba

$3^{\mathrm{a}} \mathrm{sg}$. Pte.

íma-géla (Leh)

'maíz tostado'

-gêlón (Leh)

tegét ${ }^{\text {nón }(L e h)}$

-gokón' (Leh)

mála (Pec) Ver 'trenzar'

mādakámba (Leh)

$3^{\mathrm{a}} \mathrm{sg}$. pte.

1. láua (Leh)

2. láwa $(\mathrm{Pec})$

3. láua (Sap)

lāǔa-ni (Leh)

mā-nấrmấ-no (Leh)

shilanyámba $(\mathrm{Pec})$

trosha shicón (Pec)

'entrojar'

šožimākámba (Leh)

$3^{\text {a }}$ sg. pte

(Jo 'lluvia'

ši- 'hacer') 
553. Trueno

554. Tule (junco)

555. Tumba

556. Uña

557. Urraca

558. Vaca

559. Vagina

560. Vapor

561. Vello

562. Venado

563. Vender

564. Venir

565. Venus

566. Ver

567. Verde (azul)

568. Verde (cele)

569. Verraco

570. Vestimenta

571. Vía Láctea (cf. camino)

572. Vieja

573. Viejo

574. Viento

575. Vientre

576. Viga

577. Volar

578. Volcán

579. Vosotros

580. Ya

581. Yagual

582. Yerno

583. Yo

\begin{tabular}{|c|c|}
\hline 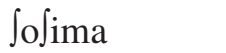 & šóžimā (Leh) \\
\hline mats'a & mắts’a (Leh) \\
\hline ora & ốra (Leh) \\
\hline kumam & 1. kúmam (Leh) \\
\hline & 2. igúman (Pec) \\
\hline ts'î́mts'ì-kātū & ts'î́mts'i-kắtšu (Leh \\
\hline waka & 1. u้ấgaš (Leh) \\
\hline & 2. wagash $(\mathrm{Pec})$ \\
\hline Jun $\int u_{N}$ & 1. šunşuN-na (Cam) \\
\hline & 2. šǔnšǔn`-na (Leh) \\
\hline
\end{tabular}

Ver Humo

Ver Cabello

ats'uwan

1. ắts'úan' (Leh)

2. ats'nan (Pec)

3. uts'uwaN (Cam)

ami-

1. amikón (Leh)

po-

siripuka

pōkón' (Leh)

sīri búga (Leh)

(lit. 'estrella grande')

jot-

ts'ahjas

yotin (Pec)

1. ts'á́xiăn' (Leh)

2. ta'áyan (Pec); probable error de a por $\underline{\mathrm{s}}$ ôsăn (Leh)

map'itkobo (Cam)

map'it kopo

Ver Ropa

k'in

Ver Anciana

koko

2. gógo (Pec)

usika

udsíga (Pec)

ū síga- (Leh)

k'omo

melan

1. mæ̂́làn (Leh)

2. mélan (Pec)

el- $\int a-$

éldžấ-okámba (Leh)

$3^{\mathrm{a}} \mathrm{sg}$. Futuro

1. érrèā (Leh)

2. erá (Pec)

kinani

af

oltom

kí-năni (Leh)

ăs̀ (Leh)

óldòm (Leh)

amte

1. ūamdé-na (Leh)

2. maiámde (Pec)

ū-ấni ú-nō (Leh) 
584. Yuca

585. Zacate

586. Zanate

587. Zancudo

588. Zapote

589. Zompopa

590. Zonchinche

591. Zonzapote

592. Zopilote

593. Zorra

594. Zorrillo

juka
Jara
ts'imts'im sinka

ts'elka

jolo

tiskute

ma apt'ut'u ÿúka (Leh)

(Prést.)

1. šara (Cam)

2. šấra (Leh)

ts'imts' i-tsínga (Leh)

(Sinka'negro')

oincii (Cam)

ts'élga (Leh)

1. yolo (Cam)

2. ÿốlo (Leh)

3. iólo (Pec)

ắnùn (Leh)

tỉn'kútè (Leh)

1. mašaptutu (Cam)

2. māžap (Leh)

3. matsáp'tsu 'tsu (la)

'zapotillo'

mayáp (Pec)

('súngano')

kuskus

1. kuskus (33am)

2. kúskús (Leh)

3. cúscus (Pec)

âu (Leh)

1. tigiš (Cam)

2. tígis (Leh)

3. tiish (Pec)

\section{Pronombres personales}

$\begin{array}{ll}\text { unani } & \text { 'yo' } \\ \text { manani } & \text { 'tú' } \\ \text { inani } & \text { 'él, ella' } \\ \text { pinani } & \text { 'nosotros, nosotras' } \\ \text { kinani } & \text { 'vosotros, vosotras' } \\ \text { maní } & \text { 'ellos, ellas' }\end{array}$

Las raíces de estos pronombres se prefijan para indicar el poseedor de un objeto o el complemento directo del verbo. 


\section{Verbo}

\subsection{Sufijos de persona del sujeto}

\begin{tabular}{|c|c|}
\hline$-\mathrm{u} \sim-\phi$ & $\begin{array}{l}1^{\mathrm{a}} \mathrm{sg} \text { ' (el alomorfo } \phi \text { se usa después del formativo de } \\
\text { presente, -u en los demás casos) }\end{array}$ \\
\hline -mi & $2^{\mathrm{a}} \mathrm{sg}$ ' \\
\hline -pa & ' $3^{\mathrm{a}} \mathrm{sg}$ ' \\
\hline$-\mathrm{pi}$ & ' $1^{\mathrm{a}} \mathrm{pl}$ ' \\
\hline$-\mathrm{ki}$ & ' $2^{\mathrm{a}} \mathrm{pl}$ ' \\
\hline -lipa & ' 3 a $\mathrm{pl}$ ' \\
\hline
\end{tabular}

3.2. Sufijos de aspecto

/-an -am- -on/ 'imperfectivo' (-on- se usa ante el alomorfo $\phi$ de la $1^{\mathrm{a}}$ persona, -am- ante bilabial y -an- en los demás casos)

\subsection{Perfectivo}

/ -aš- /

Aparentemente, no se combina con indicadores de tiempo.

\subsection{Sufijos de tiempo}

/ -ko- /

/ - - iga- /

'futuro' (se da siempre seguido por 'imperfectivo') 'pretérito' (se da siempre precedido por 'imperfectivo')

\section{Bibliografía}

Campbell, Lyle. 1976. “The last Lenca”. International Journal of American Linguistics. 42 (1)

1976. "The linguistic prehistory of the Southern Mesoamerican Periphery". Fronteras de Mesoamérica, 14a Mesa Redonda, Vol.1. Méjico: Sociedad Mexicana de Antropología.

1978. "Distant Genetic relationships and diffusion: a Mesoamerican perspective". Actas del 52 Congreso Internacional de Americanistas. París.

1979. "Middle American languages". En: Campbell, Lyle y Marianna Mithum. The languages of Native America: Historical and Comparative assessment. Austin: University of Texas Press.

Campbell, Lyle, Anne Champman y Karen Dakin. 1978. "Honduran Lencan". International Journal of American Linguistics. 44 (4). 
Chapman, Anne. 1978. Los lencas de Honduras en el Siglo XVI. Tegucigalpa: Instituto Hondureño de Antropología e Historia.

Del Río, Ximena. 1999. "El lenca de Chilanga”. Revista de Filología y Lingúística. XXV (1): 193-209.

International Phonetic Association. 1949. The principles of the International Phonetic Association. London: Department of Phonetics, University College.

Kaufman, Terrence. 1973. "Areal linguistics and Middle America”. En: Current Trends in Linguistics. Vol.II Editados por T. Sebeok the Hague: Mouton.

Lepsius, R. 1855. Das allgemeine linguistische Alphabet. Berlín: Verlag von Wilhelm Hertz.

Pike, Kenneth L. 1947. Phonemics: a technigue for reducing languages to Writing. (Twelth printing 1971). Ann Arbor: University of Michigan Press.

Schuller, Rudolf. 1928. Las lenguas indígenas de Centro América, con especial referencia a los idiomas aborígenes de Costa Rica. San José: Imprenta Nacional. 